\title{
CONTRIBUTION UF THERMAL NOISE TO THE LINE-WIDTH OF JOSEPHSON RADIATION FROM SUPERCONDUCTING POINT CONTACTS
}

\author{
A. H. Silher and J. E. Zimmerman \\ Srientific Laboratory. Ford Motor Company \\ Dearborn. Michigan \\ R. A. Kamper: \\ N.B.S. Institule for Materials Research \\ Boulder. Colorado \\ (Received 14 July 1967)
}

\begin{abstract}
The line-width of the Josephson uscillations of a voltage-biased supercor ducting point contact has been measured between $1.4^{\circ} \mathrm{K}$ and $8^{\circ} \mathrm{K}$, with bias resistors $R$ between $1.7 \times 10^{-10} \Omega$ and $2.6 \times 11^{-5} \Omega$. Within the experimental accuracy the line-wide is proportionial in $R T$. and is consietent with the estimated theoretical value $8 k T R / \Phi_{0}^{2}$. where $k$ is Boltzmann's constant and $\Phi_{\mathrm{o}}$ is the Hux quantum. Iine-widths betow $(0.1 \mathrm{~Hz}$ have been observed at 4.20 $\mathrm{K}$ for $R=1.7 \times 10^{-10} \Omega$. providing an experimental upper limit to ocher noise sources and indicating that this is useful as a voltmeter and thermometer below $1\left(1^{-16} \mathrm{~V}\right.$ and $11^{-\infty} \mathrm{K}$.
\end{abstract}

It has been proposed' that a voltage-biased superconducting point contact ${ }^{2 s}$ might be used as a low temperature thermometer. This proposal was based on the assumption that the main contribution to the line-width of the Josephson radiation from a superconducting point contact comes from the thermal noise voltage on the shunt : esistor supply-

\footnotetext{
- Supported in part by NASA.
}

ing the bias voltage. A simple argument then predicts that the line-width should be proportional to the shunt resistance $R$ and absolute temperature $T$ of this resistance. We report here an experimental verification of these predictions for values of $T$ between $1.4^{\circ} \mathrm{K}$ and $8^{\circ} \mathrm{K}$ and of $R$ between $1.7 \times$ $10^{-10} \Omega$ and $2.6 \times 10^{-8} \Omega$.

The experimental arrangement follows that previously described ${ }^{x-4}$ and is shown in Fig. I. Because

\section{Reproduced by the}


of its small resistance the shunt appears to the proint contact as a voltage source ${ }^{x}$

$$
\nu=v_{0}+\nu_{i}
$$

where $V_{0}=I_{0} R$ and $V_{s}$ is the random Huctuating component due to thermal noise. We employed two different methods to measure the line-width, referred $(0)$ as the direct and parametric methods. The direct method consisted of measuring the power output from the point contact with a $27-\mathrm{MHz}$ receiver whose bandwidth was limited to about 1 $\mathrm{kHz}$ by a quartz crystal filter as shown in Fig. $\mathrm{l}$. The bias current $l_{0}$ was scanried slowly through the value $\Phi_{0} f_{b} / R$ (where $\Phi_{0} f_{11}=2.97 \times 10^{-15} \times 27 \times$ $10^{6} \mathrm{~V}=0.56 \mu \mathrm{V}$ ) and the receiver output was recorded as a function of $I_{10}$. Since this method is limited to line-widths greater than $1 \mathrm{kHz}$ by the crystal filter and will ultimately be limited by the stability of $I_{0}$ upon further reducing the bandwidth by conventional hoterodyning. we also employed a novel parametric method as shown in Fig. 1. This method, which will be fully described in a separate publication, is related to the oscillating detector mode $^{4.5}$ previously employed and requires a 27. MHz signal to be weakly coupled into the Josephson oscillator in acddition to the $\mathrm{dc}$ bias $I_{\mathrm{b}}$. Mixing of the Josephison frequency, $f_{J}=I R / \Phi_{1}$, and the receiver frequency, $f_{10}=27 \mathrm{MHz}$, by the nonlinear behavior of the point contact produces signal at $f_{n} \pm f_{j}$. For $f_{1}$ less than the receiver bandwidth (I5) $\mathrm{kHz}$ ) the demodulated signal accurately reproduced $f_{f}$, which is then filtered via a (variable)

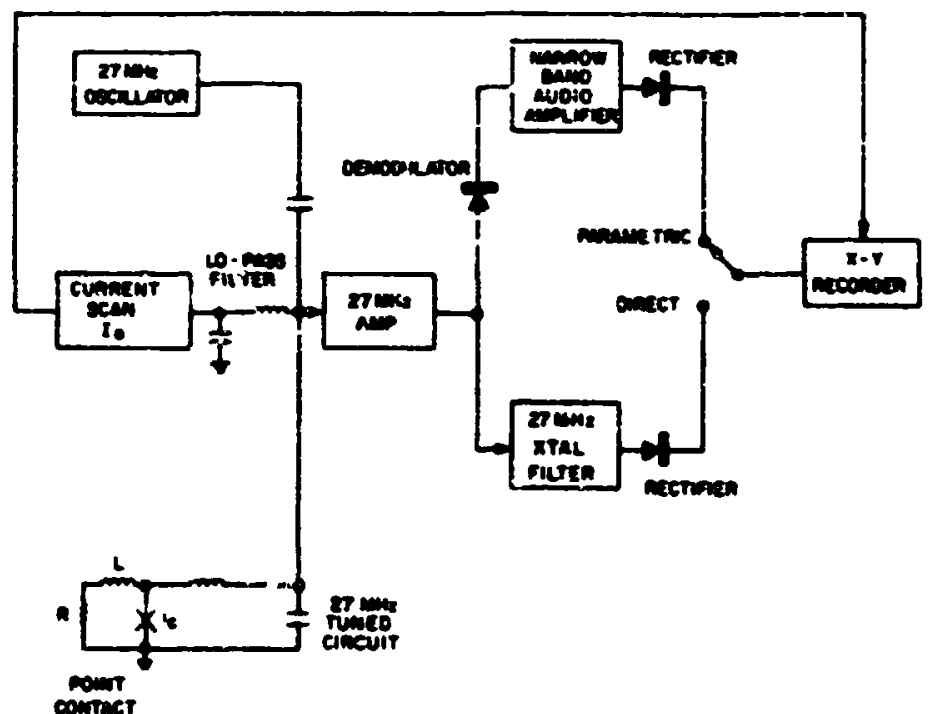

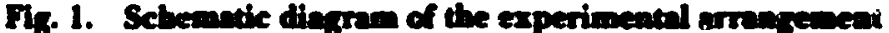
for mearurieg the joevpheon line-width. For the direct method

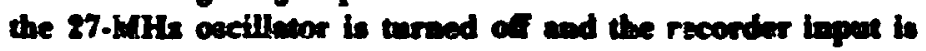
switched to the eryoul Alter channel; for the porninetric anedhod

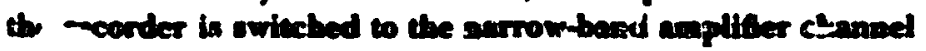

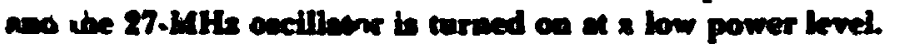

narrow-band audio amplifier. Again as in the direct method above, this filtered signal is rectified and recorded as a function of $I_{0}$. The obvious advantage of the parametric over the direct method is that it permits observation of $f$, in the audio frequency region and below, where it is relatively easy to measure line-widths of the order of $\mathrm{I} \mathrm{Hz}$ without stringent stability conditions for $l_{0}$ and the $\mathrm{rf}$ frequency-determining components.

Some results of the measured line-widths $\Delta y$ are given in Table 1. The accuracy of these data is $\pm 10 \%$. Some inconsistency is anticipated since the observed line-shapes . ary with the operating point of the if detectors. Also the signal-to-roise ratio was not sufficient to permit an accuraie line-width determination and a time-averaging method would be desirable. However, in the case of large $\Delta f$ the direct and parametric measurements give the same resilt showing that both methods measure the same quantity.

The observed temperature dependence is shown in Fig. 2 for the 26- $\mu \Omega$ resistance using the parametric method. In Fig. 2(a) a 11-khz narrow-band amplifier with a $Q=25$ was used at nominal temperatures of 2,4 , and $8^{\circ} \mathrm{K}$. In order to directly compare the line-widths, Fig. $2(b)$ shows the response where both the requency scale and the narrow-band amplifier f:equency are varied proportional to $?$. The Jusephson line-widt: from voltage-biased point contacts is shown to be linear in both $T$ and $R$ ove the ranges investigated. No evidence of line broridening by other noise sources. e.g., magnetic field tiuctuatioi., " "ceiver input noise leeding back into the cryostat, or shot noise in the junction. has been abserved in this study.

We can obtain a simple physical interpretation of these results by considering the random frequency modulution of the Josephson oscillation

\begin{tabular}{|c|c|c|c|c|}
\hline \multirow[b]{2}{*}{$\boldsymbol{R}(\boldsymbol{\Omega})$} & \multirow[b]{2}{*}{$J(x)$} & \multicolumn{3}{|c|}{ Line-widah $\Delta /(\mathrm{Hz})$} \\
\hline & & Buparin & 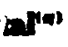 & Theory \\
\hline $2.6 \times 11^{-5}$ & $\begin{array}{l}8 \\
4 . ? \\
4.2 \\
2 \\
1 .\end{array}$ & $\begin{array}{l}8200 \\
4500 \\
3800 \\
2100 \\
1700\end{array}$ & $\begin{array}{l}P \\
D \\
P \\
P \\
D\end{array}$ & $\begin{array}{l}5350 \\
2000 \\
2800 \\
1940 \\
1140\end{array}$ \\
\hline $2.6 \times 10^{-4}$ & $\begin{array}{l}4.2 \\
4.2\end{array}$ & $\begin{array}{r}<\mathbf{i 0 0 0} \\
\mathbf{3 8 0}\end{array}$ & $\begin{array}{l}D \\
P\end{array}$ & $\begin{array}{l}280 \\
281\end{array}$ \\
\hline $1.7 \times 10^{-10}$ & 4.2 & $<0.1$ & $P$ & 0.018 \\
\hline
\end{tabular}

(a) $P$ - parametric ruet hoc!: $D$-direct method.

(1) Cakulated from Eq. (4). 
caused by thermal noise on the bias resistor. We start with Nyquist's formula

$$
\left\langle V_{N}\right\rangle=4 k T R f_{r}
$$

where $(V .8)$ is the mean square noise voltage and $f_{r}$ is the width of the band of noise to which the system is sensitive. This extends from zero frequency up to a cutoff which we estimate ty considering a single component of the noise spectrum at frequency $f_{m}$.

The spectrum of a sine wave which is fiequency modulated over a range of about a center frequency $f_{0}$ at a 1 qudation frequericy $f_{m}$ consists of an array of equally spaced side bands at interval $f$. All the side bands of significant amplitude occur in a range $\left(f_{0}-8\right)$ to $\left(f_{0}+8\right)$, so that if $f_{m}>8 f$ there are no

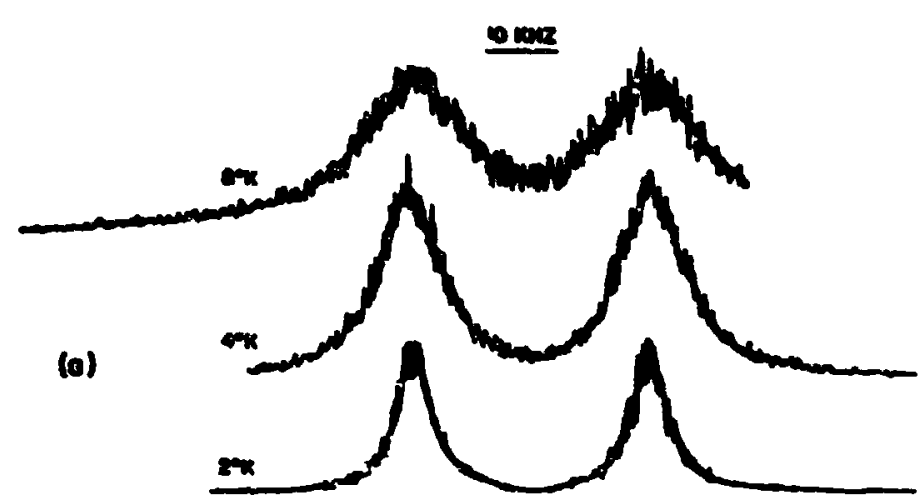

(b)

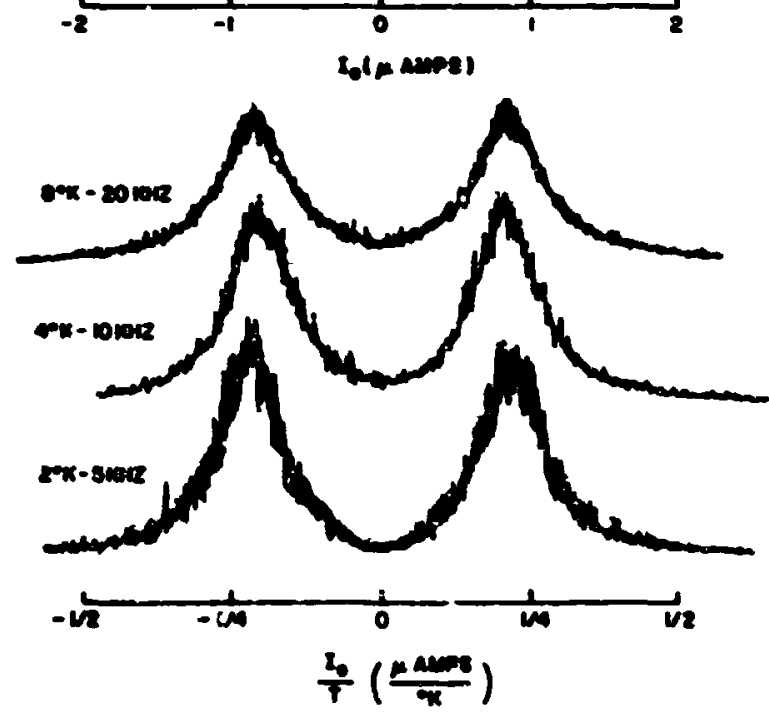

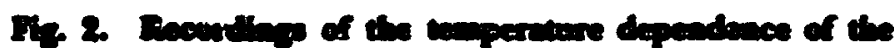

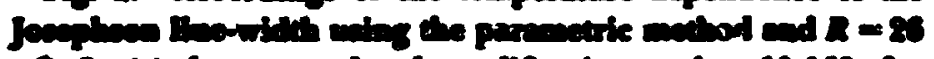

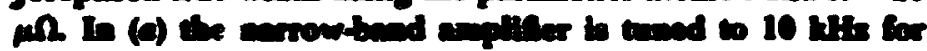

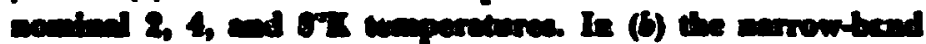

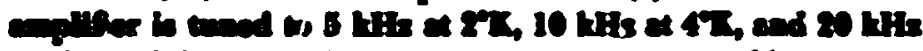

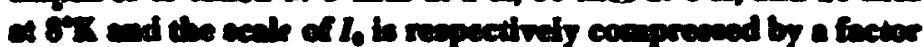
at 2. la:ge side bands and a normal receiver would not rietect the modulation. This limit corresponds physically to the variation of phase due to the modulation. This limit corresponds physically to the variation of phise due to the modulation becoming less than oue cycle.

Using the Josephson relationship between voltage and frequency we find

$$
8 f=\left(V_{N}^{2}\right)^{1 / 2} / \phi_{0}
$$

where $\Phi_{0}=h / 2 e$. Applying the condition that $f_{m}$ must not exceed of we find that the cutoff frequency $f_{c}$ in Eq. (2) is of the same order of magnitude as $\delta f$ in Eq. (3). Equating $f_{c}$ and $8 f$, combining Eqs. (2) and (3), and defining the full line-width $\Delta=28$, we have

$$
\Delta=8 k T R / \Phi_{0}^{2}=2.57 \times 10^{7} R T .
$$

Equation (4) is consistent with much more rigor. ous calculations by Scalapino" and by Burgess." It predicts correctly the onder of magmitude of the observed line-width as well as its linear deperdence on $R$ and $T$.

A corollary of the above discussion is that the line-width is independent of the frequency in agreement with the observations. In the direct method the Josephson oscillator was operated at $27 \mathrm{MHz}$, while in the parametric method it was operated at audio frequencies and below. The data given in the last line of the table were obtained by operating the Josephson oscillator at a frequency less than 1 Hz, at a bias voltage less than $10^{-15} \mathrm{~V}$. Such a device may be used as a volimeter, with sensitivity comparable to that reported for other quantum interference techniques."

'R. A. Kamper. Symponium on the Physics of Superconducting Devices, Charictesville (proccedings of this symposium are to be published an ONR report).

J. E. Zimmerman, J. A. Cowen, and A. H. Silver, Apl Phy. Leters 8,353 (1966).

'J. E. Zimmermen and A. H. Silver, 10h Incernational Conference on Low Temperature Phyrics, Moscow.

A. H. Sitver, Syrapocium on the Fhrics of Supescondycting Devices, Charbuesville (see ref. 1).

A. H. Silver and J. E. Zimmerman. Apph Phy. Lntws IS, 142 (1967).

-D. J. Scalapino, Sympoeium on the Phyica of Superconducting Devices, Ct arbucerville (cee ref. 1).

'R. E. Burgeses, Sympotium on the Physica of Superconducting Devices, Charbotesvill. (see ref. 1).

J. Carte, Phil. Mag. 13, 115 (1966). 